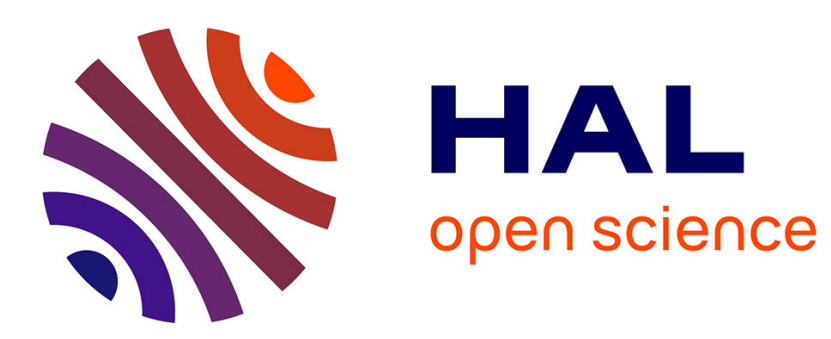

\title{
CARACTÉRISATION ULTRA-ACOUSTIQUE DE RÉSEAUX POLYURÉTHANE
}

\author{
J. Emery, M. Tabellout, H. Randrianantoandro, Denys Durand
}

\section{To cite this version:}

J. Emery, M. Tabellout, H. Randrianantoandro, Denys Durand. CARACTÉRISATION ULTRAACOUSTIQUE DE RÉSEAUX POLYURÉTHANE. Journal de Physique IV Proceedings, 1992, 02

(C1), pp.C1-775-C1-778. 10.1051/jp4:19921169 . jpa-00251129

\section{HAL Id: jpa-00251129 \\ https://hal.science/jpa-00251129}

Submitted on 1 Jan 1992

HAL is a multi-disciplinary open access archive for the deposit and dissemination of scientific research documents, whether they are published or not. The documents may come from teaching and research institutions in France or abroad, or from public or private research centers.
L'archive ouverte pluridisciplinaire HAL, est destinée au dépôt et à la diffusion de documents scientifiques de niveau recherche, publiés ou non, émanant des établissements d'enseignement et de recherche français ou étrangers, des laboratoires publics ou privés. 


\title{
CARACTÉRISATION ULTRA-ACOUSTIQUE DE RESEAUX POLYURÉTHANE
}

\author{
J.R. EMERY, M. TABELLOUT, H. RANDRIANANTOANDRO et D. DURAND \\ Faculté des Sciences U.R.A., CNRS 807 et 509, Avenue O. Messiaen, BP. 535, F-72017 Le Mans \\ cedex, France
}

\section{Resumé}

La formation et les propriétés finales de réseaux macromoléculaires covalents sont étudiés au moyen de techniques de propagation ultrasonore. Dans le cas de réseaux polyuréthane, l'évolution de la vitesse de propagation et de l'absorption des ondes longitudinales montrent l'interêt de la spectroscopie ultrasonore utilisée comme méthode d'investigation de la croissance du réseau ainsi que des propriétés du matériau à l'état achevé.

$1-$

\section{INTRODUCTION}

Dans le cadre général de l'étude des propriétés des réseaux covalents et de leur relations structure-propriétés, les réseaux polyuréthane présentent un double interêt: sur un plan fondamental, ils sont très étudiés et bien décrits par les théories actuelles de croissance des réseaux qui rendent compte d'une manière satisfaisante de leurs propriétés, sur un plan pratique, c'est un matériau très utilisé et d'application multiple.

La détermination des modules élastiques, dans une gamme de fréquence aussi large que possible, présente un interêt fondamental certain pour la description des propriétés mécaniques du matériau, tant au cours de la réaction de réticulation que pour le réseau final. Il est donc essentiel de pouvoir appréhender ces grandeurs en temps réel au fur et à mesure de la formation du gel de polyuréthane en suivant à chaque étape la dépendance en fréquence des modules élastiques. Ainsi la technique de spectroscopie ultrasonore a été appliquée à la caractérisation de réseaux polyuréthane dans leur phase d'élaboration et dans leur état final.

II-

\section{DISPOSITIF EXPERIMENTAL}

Une impulsion ultrasonore est expédiée dans le milieu étudié et le signal reçu est numérisé et analysé dans le domaine fréquentiel pour déterminer la fonction de transfert $H^{*}(\mathfrak{f})$ complexe du matériau. Cette fonction est la transformée de Fourier de la réponse impulsionnelle $h^{*}(t)$ (rapport du signal de sortie sur le signal d'entrée) et décrit 
entièrement les propriétés mécaniques du milieu dans lequel elle se propage, ce domaine est limité par les bandes passantes des différents composants du dispositif, et principalement par celles des traducteurs. [1]

Expérimentalement, le signal ultrasonore reçu est amplifié et numérisé au moyen diun oscilloscope numérique. Le micro ordinateur effectue la transformée de Fourier discrète et peut ainsi determiner la fonction de transfert du système. Le temps de mesure depend donc du temps d'acquisition, de la fréquence de mesure et du moyennage du signal. Il évolue donc de quelques millisecondes à quelques secondes.

Le matériau étudié est placé dans une cellule thermostatée dont l'entrefer est réglable de $\mathrm{O}$ à quelques centimètres.

Ce dispositif est utilisé pour le suivi des propriétés élastiques des réseaux macromoléculaires et des gels [2]. Dans le cas du polyuréthane, seules les ondes longitudinales se propagent et le paramètre expérimentalement mesurable est le module longitudinal $\mathrm{M}^{*}$. En effet, la propagation des déplacements longitudinaux $\mathrm{X}$ dans la direction $\mathrm{x}$ est régie par l'équation de la forme:

$$
\left(\partial^{2} \mathrm{X} / \partial \mathrm{t}^{2}\right)=\mathrm{M}^{*} / \rho\left(\partial^{2} \mathrm{X} / \partial \mathrm{x}^{2}\right) \quad \text { ( } \rho \text { masse volumique du matériau) }
$$

Les parties réelles et imaginaires de $M^{*}$ s'expriment en fonction de la vitesse $V$ et de l'absorption $\alpha$ :

$$
\begin{array}{ll}
M^{\prime}=\rho V^{2}(\omega)=\left(K^{\prime}+4 / 3 G^{\prime}\right) & K: \text { module de compressibilité } \\
M^{\prime \prime}=2 \rho V^{3} \alpha(\omega) / \omega=\left(K^{\prime \prime}+4 / 3 G^{\prime \prime}\right) & G: \text { module de cisaillement }
\end{array}
$$

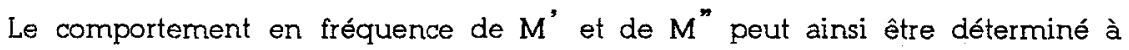
partir de la mesure du comportement en fréquence de $V$ et de $\alpha$, ce que permet la technique de spectroscopie ultrasonore dans la limite de la bande passante autorisée.

\section{III- $\quad$ SYSTEME ETUDIE}

Le Polyuréthane étudié est obtenu par polycondensation de Polyoxypropylènetriol $(\mathrm{M}=\mathrm{T} O \mathrm{O})$ avec l'hexamethylène diisocyanate (HMDI) selon des conditions experimentales définies ailleurs.[3]

$$
\mathrm{C}_{2} \mathrm{H}_{5}-\mathrm{C}\left[\left[\mathrm{E}\left(\mathrm{OCH} \mathrm{CHCH}_{3}\right)_{n}\right]-\mathrm{OH}\right]_{3}+\mathrm{OCH}-\left(\mathrm{CH}_{2}\right)_{6}-\mathrm{HCO}
$$

\section{IV- $\quad$ RESULTATS EXPERIMENTAUX}

Les figure 1 et 2 montrent respectivement les variations de l'absorption pour des fréquences comprises entre 6 et $36 \mathrm{MHz}$, et de la vitesse de propagation ultrasonore à $20 \mathrm{MHz}$ en fonction du temps.

L'évolution de ces signaux, comparée à d'autres méthodes d'investigation, montrent qu'ils sont sensibles au degré d'avancement de la réaction et tend vers une limite pour un taux d'avancement qui tend vers 1. 


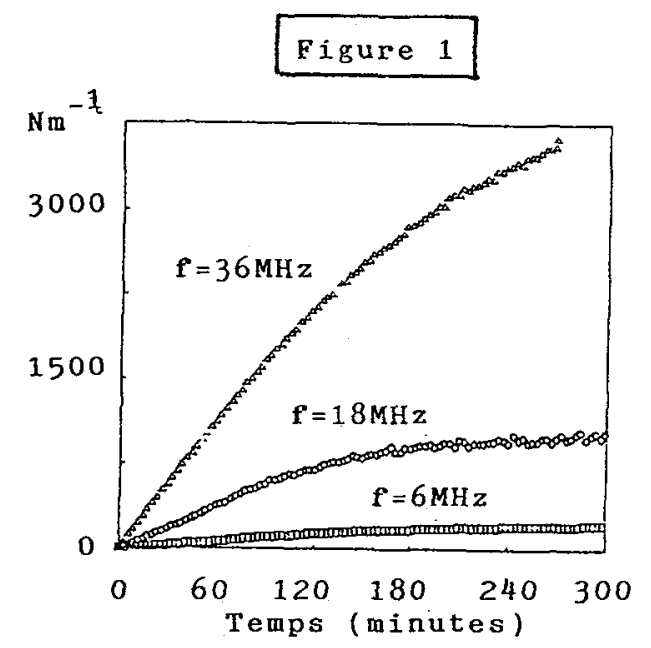

Variation de $I^{\prime}$ absorption ultrasonore au cours de la réticulation

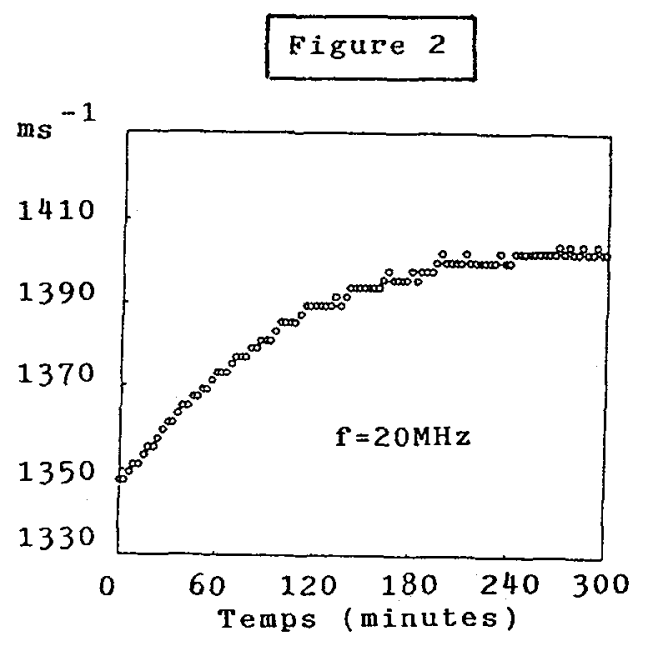

Evolution de la vitesse de propagation ultrasonore au cours de la réticulation

Figure 3

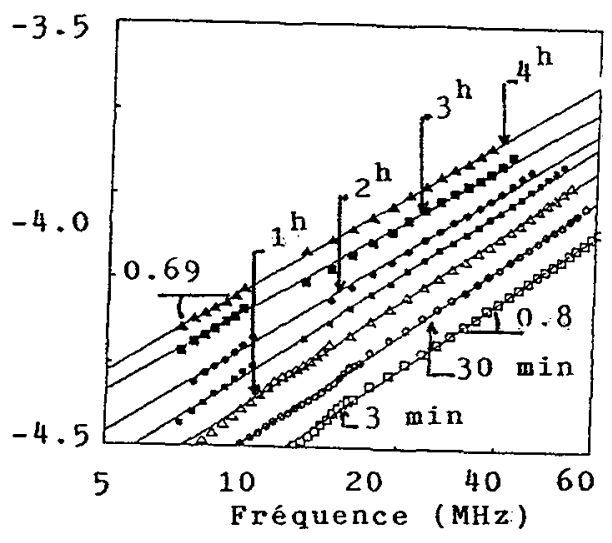

$\log (\alpha / f)$ en fonction de $\log f$

Figure 4

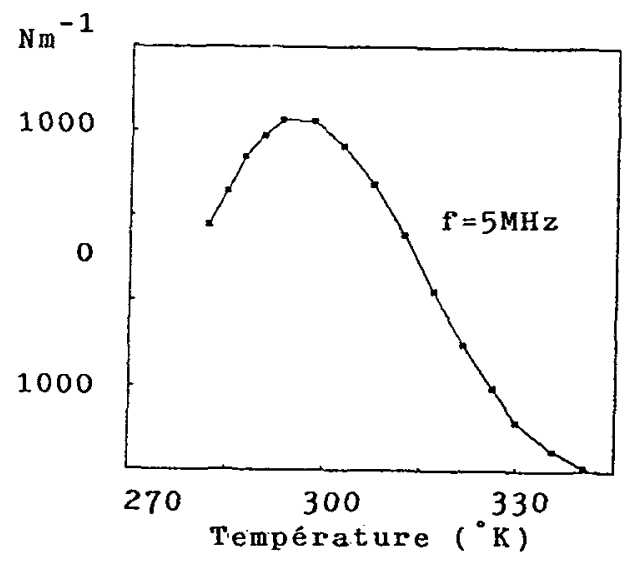

Variation de l'absorption ultrasonore en fonction de la température 


\section{V- DISCUSSION}

Schématiquement, on peut considérer que dans cette fenêtre fréquentielle on rencontre deux processus au cours de la croissance du réseau: la transition Sol-Gel liée aù formation d'un amas géant et la vitrification. Pour le premier processus il peut être montré que la dépendance en fréquence des modules élastiques de cisaillement $G^{*}$ évolue selon une loi de puissance en fréquence:

$$
G^{*} \sim G^{\prime} \sim \omega^{n}
$$

où $\mathfrak{n}$ est un exposant critique pouvant présenter un caractère d'universalité. Si on suppose que le module longitudinal $M$ suit le même comportement, la représentation Log-Log de $M^{\prime \prime}$ en fonction de $\omega$ doit être linéaire. La figure 3 montre qu'il en est ainsi et l'exposant $n$ varie de 0.80 au début à 0.69 à la fin de la réaction. Dans le domaine des fréquences rhéologiques où on est sensible uniquement au processus de gélification, l'exposant $\mathbf{n}=0.71$ correspond à la prédiction du modèle de percolation. [4]

Le phénomène de vitrification correspond à une transition Liquide-Solide et depend de la fréquence à laquelle il est observé: il se traduit par un processus de relaxation de grande amplitude. A la température d'étude, la fenêtre fréquentielle autorisée par le dispositif ne rend pas compte de cette transition, ceci signifie que le processus n'est observable à la fréquence d'étude que pour une température inférieure.

La figure 4 montre l'évolution de l'absorption ultrasonore en fonction de la température à $5 \mathrm{MHz}$. Le pic observé est attribué à cette transition.

\section{VI- $\quad$ CONCLUSION}

Cette étude sur le polyuréthane confirme que, d'une manière générale, la spectroscopie ultrasonore peut être une méthode très utile de contrôle non destructif des processus de réticulation. Elle permet de determiner des modules élastiques en temps réel dans un domaine de fréquence où ils sont sensibles aux phenomènes de gélification et de vitrification.

\section{Bibliographie}

[1] EMERY. J.R., TABELLOUT. M. Revue Phys. Appl. 25 , 243, 1990

[2] EMERY. J.R.

Annales des composites $1-2,89,1986$

[3] ADAM. M. , DELSANTI. M., DURAND. D. Macromolecules, 18, 2285, 1985

[4] LAIREZ. D. , DURAND. D. , EMERY. J. R. J. Phys. II , 1 , 9T7, 1991 